\title{
Designing Backpacks for High Fidelity Mobile Outdoor Augmented Reality
}

\author{
Wayne Piekarski, Ross Smith, and Bruce H. Thomas \\ Wearable Computer Laboratory \\ School of Computer and Information Science \\ University of South Australia \\ Mawson Lakes, SA, 5095, Australia \\ wayne@cs.unisa.edu.au,ross@tinmith.net,bruce.thomas@unisa.edu.au
}

\begin{abstract}
This paper presents the design for our latest backpack to support mobile outdoor augmented reality, and how it evolved from lessons learned with our previous designs. We present a number of novel features which help to reduce size and weight, improve reliability and ease of configuration, and reduce CPU usage on laptop computers.
\end{abstract}

\section{Introduction}

In this paper, we present our latest system for outdoor AR, named Tinmith Backpack 2004. This system replaces the previous Tinmith-Endeavour designed in 2002, which replaced the original designs we developed in 1999. The goal of our work is to support experimental outdoor AR research, such as our $3 \mathrm{D}$ modelling system Tinmith-Metro [3]. We have been designing backpacks since 1998 in a similar spirit to various others [1] [2]. We require both video and optical overlay AR to be performed on a mobile platform, and currently use the following devices: Dell Inspiron 8100 laptop; Trimble Ag132 GPS or Trimble 4700 RTK GPS; InterSense InertiaCube2; IO-Glasses PAL, IO-Glasses SVGA, or Sony Glasstron PLM-S700e.

\section{Design rationale}

The backpack shown in (1) of Figure 1 is one of our original designs which contained all the equipment chaotically strapped to a hiking frame. Changes were difficult to make because everything was buried under the cables, and there were frequent failures due to mechanical stresses.

The Tinmith-Endeavour 2002 design shown in (2) of Figure 1 was designed in collaboration with engineers from the Defence Science Technology Organisation ${ }^{1}$ to be flexible for future changes and to protect the equipment inside. After using this design for two years, we have now learned that the design was in fact too flexible - the polycarbonate box was very large for future expansion and always had spare room inside it. With constant changes in technology, we gradually upgraded the internal devices which tended to get smaller over time. By keeping the design flexible the cost was extra size and weight, which reduced portability and comfort.

In the new 2004 design shown in (3) of Figure 1, we have committed ourselves to a certain range of devices so

\footnotetext{
${ }^{1}$ Information Technology Division / Scientific and Engineering Services Defence Science Technology Organisation, Salisbury, South Australia, 5108
}

that we can keep the footprint as small as possible. Rather than add large interface boxes, we have modified the hardware to use the interfaces desired. Instead of leaving room for future expansion that may not occur, we have designed the backpack to fit what we require today. The result is dimensions of $37 \mathrm{~cm} \mathrm{(w)} \mathrm{x} 30 \mathrm{~cm}$ (h) $\times 8 \mathrm{~cm}$ (d) which is much smaller than the previous $40 \mathrm{~cm}$ (w) x 54 $\mathrm{cm}(\mathrm{h}) \times 15 \mathrm{~cm}$ (d) - a reduction of more than a factor of three in volume.

\section{Frame design}

The core of the 2002 backpack was a polycarbonate box mounted onto a breathing apparatus frame. This design allowed the backpack to be unfolded on the ground and the laptop opened out like a table for easy debugging. While this feature was nice, it added considerable extra weight and further thickness to the polycarbonate box. Our new polycarbonate box is smaller and attaches tightly to a modified baby carrying harness, which is much more comfortable and very lightweight. Since the hinge is not required the backpack does not need as much structure to support it, giving further reductions in size and weight.

\section{Attachments}

Rigidly attaching devices is difficult since many of them come with no mounting points or area to drill in to. We have tested a variety of strategies to fix devices down securely in previous designs: straps do not hold down firmly enough, while cable ties, double sided tape, and bolts are not quickly removable.

For attaching most devices, we have found that $3 \mathrm{M}$ DualLock Velcro is the most versatile. This Velcro has mushroom shaped plastic heads, and four $1 \mathrm{~cm}^{2}$ pieces can

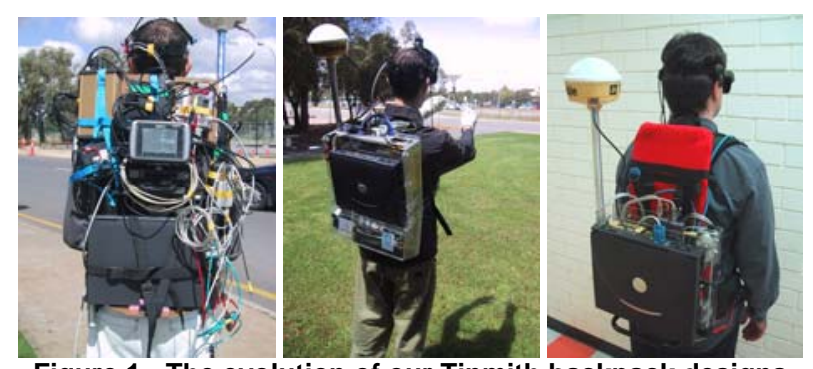

Figure 1 - The evolution of our Tinmith backpack designs (1) Unorganised design from 2001 with straps on hiking frame (2)Tinmith-Endeavour from 2002 with polycarbonate casing (3) New Tinmith backpack 2004 with compact box and frame 
securely suspend a laptop vertically. For extra safety, the externally exposed laptop is held using straps as a fail safe, since we noticed that over time the Velcro may wear out and its effectiveness reduces. Devices inside the backpack also use Velcro, and do not require any safety catches because they are light in weight and the surrounding devices and cables will support the device. Using this Velcro allows us to snap devices in and out very quickly, which is useful when devices need to reconfigured or used for other projects.

The Velcro with pads and mushroom heads provides about $4 \mathrm{~mm}$ of clearance under devices attached to the polycarbonate box. This clearance is used to route many of the internal cables underneath the devices between the pads, which prevents cable movements. Velcro cable ties are used to route cables along the outside piping where there are no flat surfaces.

\section{Data and power cabling}

The devices we integrate use a variety of power and data cables. Many devices still use RS-232, which uses rather large DB9 connectors. Since most laptops contain only a single RS-232 port, a PCMCIA or USB to RS-232 break out device is also needed to integrate many devices. Rather than using separate power and RS-232 cables for each device, we have decided to embed FTDI 232BM ${ }^{2}$ USB to RS-232 converters into any device if possible. With an integrated USB chip, a single USB cable can provide both power and data and with smaller connectors. For devices that need only power, we use USB cables to provide the necessary 5V voltage. Using USB for all RS-232 and $5 \mathrm{~V}$ supplies, the internals are simplified significantly.

The new power supply operates using $12 \mathrm{~V}$ NiMH batteries rated at $100 \mathrm{Wh}$, with compact DC to DC converters providing regulated $12 \mathrm{~V}$ and $5 \mathrm{~V}$ outputs. The $5 \mathrm{~V}$ supply is provided with $1.3 \mathrm{~mm}$ sockets to the USB hubs which are responsible for distributing power to any $5 \mathrm{~V}$ devices. The $12 \mathrm{~V}$ supply is available from $2.5 \mathrm{~mm}$ sockets, for devices such as the GPS and Firewire hub. The InertiaCube2 needs $6 \mathrm{~V}$ at low current so we use a simple linear regulator to supply it. In our previous 2002 system, we had a central power supply with $5 \mathrm{~V}, 9 \mathrm{~V}$, and $12 \mathrm{~V}$ all available from very large 4 pin connectors. These connectors wasted a lot of space to provide unused flexibility.

The 2002 backpack contained four USB sockets, the glove sockets, HMD connectors, and a power switch on the shoulder straps for easy access. We found that these got in the way when untrained users were putting on the backpack, with possible damage to the cables over time. We also found that it was hard to see the ports while wearing the backpack. Our new design places these connectors onto the waist straps, with improved visibility and the ability to leave external devices on the floor while putting the backpack on. The 2002 design required an assistant to hold the devices because the cables were not long enough to go to the shoulders. Only the glove connectors are on the shoulders now, and these are kept flush with the straps to not get in the way.

\section{High performance AR overlay}

The recent trend in outdoor AR backpacks has been the use of Firewire or USB cameras for laptops to perform real-time video tracking and video overlay. Rather than using a laptop to process this video (burdening the CPU/GPU and using excessive power) we have recently started using specialised hardware to perform this task. We currently use a small Grandtec MagicView ${ }^{3}$ video overlay unit which combines VGA images on a black background with composite camera video to provide the output for the HMD. The video overlay is performed at full PAL refresh rates with a power consumption of only $1.9 \mathrm{~W}$. We have also been investigating the use of reconfigurable computers with field programmable gate arrays to implement our hand tracking algorithms with power consumption of only $4.8 \mathrm{~W}$. The laptop does not handle any video now, with improved frame rates and power consumption, and the system still provides both video and optical AR overlay.

\section{Conclusion}

This paper has presented our latest ideas for the design of backpacks for high fidelity mobile AR, based on experience gained through iterative design steps. Using a design with reduced flexibility, the overall size and weight are greatly reduced. The polycarbonate box provides protection for the devices as well as a flat mounting surface. A baby carrying harness permits easy wearing of the device with little extra weight added. Plastic Velcro is used to permit simple reconfiguration of devices and provides a cable routing infrastructure. USB is embedded into devices to remove the need for legacy interfaces, and provides a compact power and data distribution mechanism. Finally, specialised video overlay units and FPGAs are used to perform CPU intensive tasks with minimal power consumption and faster performance.

\section{References}

[1] Feiner, S., MacIntyre, B., and Hollerer, T. A Touring Machine: Prototyping 3D Mobile Augmented Reality Systems for Exploring the Urban Environment. In 1st Int'l Symposium on Wearable Computers, Cambridge, Ma, Oct 1997.

[2] Julier, S., Lanzagorta, M., Baillot, Y., Rosenblum, L., Feiner, S., and Hollerer, T. Information Filtering for Mobile Augmented Reality. In 3rd Int'l Symposium on Augmented Reality, pp 1-10, Munich, Germany, Oct 2000.

[3] Piekarski, W. and Thomas, B. H. Interactive Augmented Reality Techniques for Construction at a Distance of 3D Geometry. In 7th Int'l Workshop on Immersive Projection Technology / 9th Eurographics Workshop on Virtual Environments, Zurich, Switzerland, May 2003.

\footnotetext{
${ }^{2}$ Future Technology Devices Intl Ltd, FT232BM chip, http://www.ftdichip.com

${ }^{3}$ GrandTec, MagicView, http://www.grandtec.com/magicview.htm
} 